Alois Kufner, Mathematical Institute, Academy of Sciences of the Czech Republic, Žitná 25, 11567 Praha, Czech Republic,

e-mail: kufner@@earn.cvut.cz

\title{
A PROPERTY OF GREEN'S FUNCTION
}

For $k$ a positive integer, let

$$
M_{i} \subset\{0,1, \ldots, k-1\}, \quad i=0,1
$$

with $M_{i} \neq \emptyset$ (i.e. $\# M_{i}>0$ ) and

$$
\# M_{0}+\# M_{1}=k
$$

where $\# M_{i}$ is the cardinality of $M_{i}$.

Suppose that the solution $u=u(x)$ of the simple boundary value problem

$$
\begin{array}{rlll}
u^{(k)}=f & \text { in } & (0,1), \\
u^{(i)}(0)=0 & \text { for } & i \in M_{0}, \\
u^{(j)}(1)=0 & \text { for } & j \in M_{1}
\end{array}
$$

with $f$ not changing sign in $(0,1)$ can be expressed uniquely in the form

$$
u(x)=\int_{0}^{x} K_{1}(x, t) f(t) d t+\int_{x}^{1} K_{2}(x, t) f(t) d t .
$$

Problem: Prove that there exist positive constants $c_{1}, c_{2}$, and nonnegative integers $\alpha_{1}, \alpha_{2}, \beta_{1}, \beta_{2}, \gamma_{1}, \gamma_{2}, \delta_{1}, \delta_{2}$, such that the following estimates hold:

$$
c_{1} \leq \frac{K_{i}(x, t)}{x^{\alpha_{i}}(1-x)^{\beta_{i}} t^{\gamma_{i}}(1-t)^{\delta_{i}}} \leq c_{2}
$$

for $0<t<x<1$ if $i=1$ and for $0<x<t<1$ if $i=2$, and determine the values of $\alpha_{1}, \ldots, \delta_{2}$.

Key Words: Green's function, Hardy's inequality

Mathematical Reviews subject classification: Primary: 34B27; Secondary: 26D10

Received by the editors Aug 9, 1995 


\section{Remarks:}

(i) The problem is solved in [1] under the additional assumption

$$
\left.M_{0} \cap M_{1}=\emptyset \quad \text { (i.e. } M_{1}=\{0,1, \ldots, k-1\} \backslash M_{0}\right) .
$$

(ii) The solution of this problem allows one to formulate necessary and sufficient conditions for the validity of the $k$-th order Hardy inequality

$$
\left(\int_{0}^{1}|u(t)|^{q} w_{0}(t) d t\right)^{1 / q} \leq C\left(\int_{0}^{1}\left|u^{(k)}(t)\right|^{p} w_{k}(t) d t\right)^{1 / p}
$$

for functions $u$ satisfying condition (2).

\section{References}

[1] A. Kufner, Higher order Hardy inequalities, Bayreuth. Math. Schriften, 44 (1993), 105-146. 